\title{
Cesarean Section for Placenta Previa and Placenta Previa Accreta Spectrum
}

\author{
Satoru Takeda, MD, $\mathrm{PhD}^{1,2}$ Jun Takeda, MD, $\mathrm{PhD}^{1}$ Shintaro Makino, MD, $\mathrm{PhD}^{1}$ \\ ${ }^{1}$ Department of Obstetrics and Gynecology, Faculty of Medicine, \\ Juntendo University, Tokyo, Japan \\ 2 Aiiku Research Institute for Maternal, Child Health and Welfare, \\ Imperial Gift Foundation Boshi-Aiiku-Kai, Tokyo, Japan \begin{abstract}
Obstetrics and Gynecology, Faculty of Medicine, Juntendo University, 2-1-1, Hongo, Bunkyo-ku, Tokyo 113-8421, Japan (e-mail: stakeda@juntendo.ac.jp).
\end{abstract} \\ Address for correspondence Satoru Takeda, MD, PhD, Department of
}

Surg J 2020;6(suppl S2):S110-S121.
Abstract Keywords
- balloon tamponade
- cesarean section
- compression sutures
- hysterectomy
- placenta previa
- placenta accreta spectrum

According to the increase in the rate of cesarean section and the increase of high-aged pregnancy, we seem to more often encounter cases with placenta previa and placenta previa accrete spectrum. There are concerns about these cases, such as difficulty in controlling bleeding from the separation surface of placenta previa, the need for hysterectomy as a life-saving procedure, systemic management and hemostasis during massive hemorrhage, and treatment of disseminated intravascular coagulation (DIC). These cases are most frequently associated with cesarean hysterectomy.
In placenta previa cases, abundant blood flow enters the uterus not only from the internal iliac artery but also via anastomosis of the external iliac artery, interior mesenteric artery, lumbar artery, median sacral artery, etc. Therefore, it is difficult to control bleeding; arterial ligation exerts a poor hemostatic effect. It is necessary to understand various hemostatic procedures, damage control surgery and resuscitation for massive hemorrhage, and systemic management. Placenta increta and percreta are diagnosed by ultrasonography, Doppler ultrasonography, magnetic resonance imaging (MRI), and cystoscopy, etc. However, it is difficult to obtain a definitive diagnosis of placenta accreta.

In cases of placenta previa with previous cesarean section, the operation should be performed in a tertiary medical facility with sufficient staff and blood available for transfusion. Preoperative placement of intra-arterial balloon occlusion catheter in the common iliac artery or the aorta is useful for the prevention of massive hemorrhage. In cases with placenta previa accrete spectrum, procedures following cesarean section include hysterectomy, preservation of the uterus with hemostasis of the placental separation surface, conservative treatment while leaving the placenta in situ, and delayed hysterectomy. Massive hemorrhage occurs at the placental separation surface (spontaneous separation and manual separation) and around the bladder (placenta percreta and placenta increta).

\section{Cesarean Section for Placenta Previa}

\section{Surgical Steps}

1. Laparotomy $\downarrow$

2. Incision in the peritoneum of the vesicouterine pouch and separation of the bladder $\downarrow$

3. Transverse incision on the uterine lower segment or the lower uterine corpus $\downarrow$

4. Delivery of the fetus $\downarrow$

5. Separation of the placenta and uterotonic medication $\downarrow$

6. Hemostasis of the placental separation surface and myometrial suture $\downarrow$

7. Abdominal closure
DOI https://doi.org/ 10.1055/s-0039-3402036. ISSN 2378-5128.

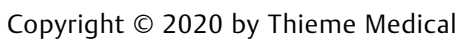
Publishers, Inc., 333 Seventh Avenue, New York, NY 10001, USA. Tel: +1(212) 760-0888.

\section{License terms} (c) $(1) \$$ 


\section{Tips and Warnings}

\section{Risk Factors for Placenta Accrete Spectrum}

Placenta previa with a prior cesarean section is a high risk for placenta accreta spectrum ( - Fig. 1; placenta accreta, increta, and percreta), that encompasses clinical adherence and pathological findings, and a major factor contributing to maternal death. ${ }^{1,2}$ Along with the increase in the rate of cesarean section, the incidence of placenta accreta spectrum increased to 1 out of 2,500 deliveries in 1997 (10-fold compared with that 50 years ago). ${ }^{3}$ The frequency of placenta accreta spectrum in cases of placenta previa is generally 1 out of 2,065 cases, but it increases to $25 \%$ in cases with one prior cesarean section and $40 \%$ in cases with two prior cesarean sections. ${ }^{4}$ According to the report by Sumigama et $\mathrm{al}^{5}$ in Japan, the frequency of placenta accreta spectrum in placenta previa is $1.1 \%$ in cases with a first cesarean section, $37.8 \%$ in cases with one prior cesarean section, and $38.5 \%$ in cases with two prior cesarean sections. In our institution, the corresponding frequency was $41 \%$ for cases with one prior cesarean section and $54 \%$ for those with two prior cesarean sections. ${ }^{1}$

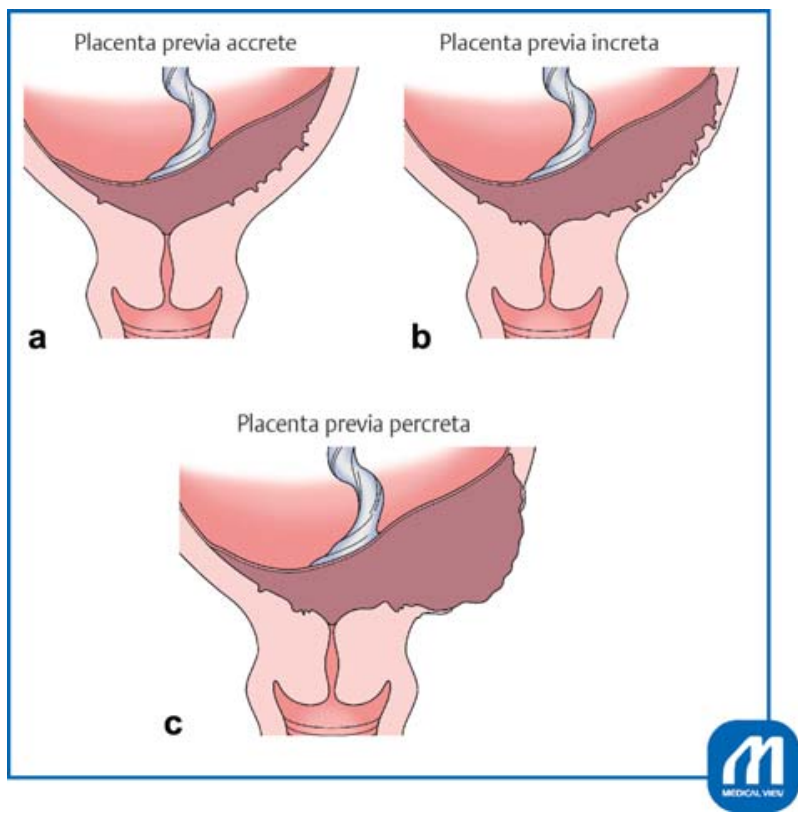

Fig. 1 Pathological definition of placenta previa accreta spectrum. (a) Placenta previa accrete. Chorionic villi directly attached to the uterine myometrium with complete or partial absence of the decidua basalis in a case with placenta previa. Those conditions can be classified as total, partial, or focal adherence of the placenta by the clinical and pathological findings abutting the myometrium. (b) Placenta previa increta. Chorionic villi invade deeply into the myometrium layer in a case with placenta previa. (c) Placenta previa percreta. Chorionic villi penetrate myometrium into the uterine serosa and the bladder, or perforate the uterine wall into the pelvic cavity in a case with placenta previa. (Reproduced with permission from Takeda S. Cesarean section for placenta previa and placenta previa accrete spectrum. In: Hiramatsu Y, Konishi I, Sakuragi N, Takeda S, eds. Mastering the Essential Surgical Procedures OGS NOW, No.3. Cesarean section. (Japanese). Tokyo: Medical View; 2010:102-115. Copyright ( ) Medical View).

\section{Preoperative Evaluation}

The state of the cervical os, fetal presentation, position of the presentation, and placental location should be confirmed by transvaginal and transabdominal ultrasonography prior to cesarean section. Placenta previa cases, particularly those with previous cesarean section, should be examined for placenta increta and placenta percreta by ultrasonography, colorDoppler ultrasonography, MRI, cystoscopy, etc. (-Figs. 2-4, - Table 1). ${ }^{1,2,6-12}$ Diagnostic imaging of placenta accreta can create many false-positive results, yielding a low rate of accurate diagnosis. It is, furthermore, difficult to obtain a definitive diagnosis of partial placenta accreta. ${ }^{1}$

\section{Tips and Warnings}

\section{Management of Placenta Previa Accrete Spectrum}

Placenta previa increta or percreta and suspected placenta previa accreta (such as in cases of placenta previa with prior cesarean section) should be managed and undergo cesarean section in a tertiary medical facility. ${ }^{1-4,6}$ In cases of suspected placenta accreta spectrum, the obstetrician should have a good knowledge of pelvic anatomy, obstetric hemostasis and damage control

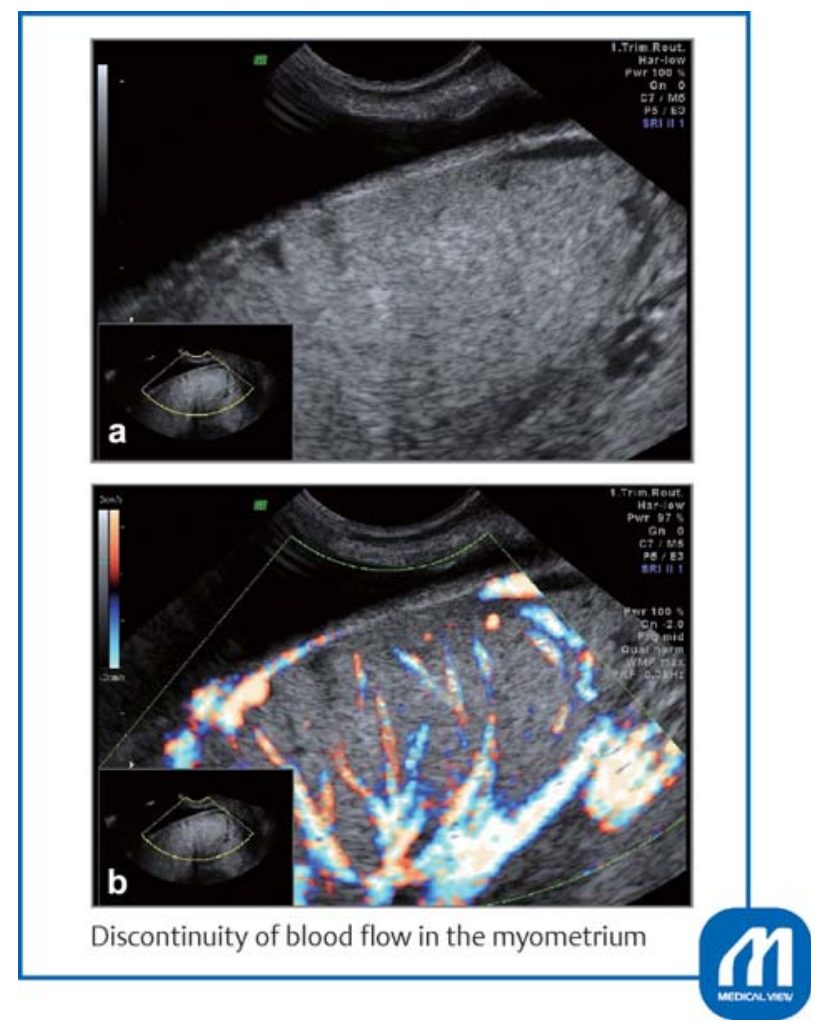

Fig. 2 Findings of placenta increta by ultrasonography. A diagnosis of placenta increta was obtained based on thinning of the myometrium, disappearance of the sonolucent zone (a) and discontinuity of the blood flow in the myometrium (b). (Reproduced with permission from Takeda $\mathrm{S}$. Cesarean section for placenta previa and placenta previa accrete spectrum. In: Hiramatsu Y, Konishi I, Sakuragi N, Takeda S, eds. Mastering the Essential Surgical Procedures OGS NOW, No.3. Cesarean section. (Japanese). Tokyo: Medical View; 2010:102-115. Copyright @ Medical View). 


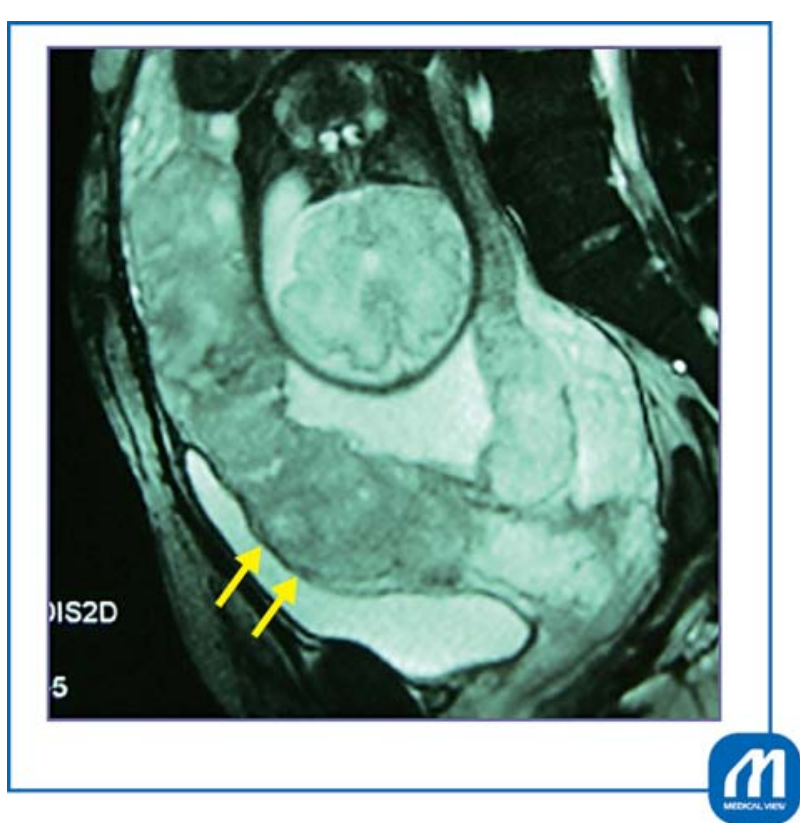

Fig. 3 MRI findings of placenta previa increta. The placenta is observed to invade the bladder from the anterior wall of the uterus. (Reproduced with permission from Takeda S. Cesarean section for placenta previa and placenta previa accrete spectrum. In: Hiramatsu Y, Konishi I, Sakuragi N, Takeda S, eds. Mastering the Essential Surgical Procedures OGS NOW, No.3. Cesarean section. (Japanese). Tokyo: Medical View; 2010:102-115. Copyright (c) Medical View).

surgery for massive hemorrhage, and he should also be skilled in performing total hysterectomy. In each case of placenta previa with/without findings of placenta accrete spectrum, the operators should discuss about approaches of cesarean delivery, management for placenta accrete spectrum, selection of treatment and prevention of massive hemorrhage, and therefore, should make a final decision of surgical strategies for placenta previa accrete spectrum (-Fig. 4). ${ }^{1}$ Preoperative preparation, including securing of staff, an anesthesiologist, and sufficient blood for transfusion, is important in cases undergoing cesarean section for placenta previa.

\section{Preoperative Preparation}

It is necessary to make sure that the patient and family understand placenta previa and placenta previa accreta spectrum, to obtain their consent for possible blood transfusion and that they comprehend the risks of massive hemorrhage and hysterectomy. Autologous blood should be stored preoperatively to prevent allogeneic blood transfusion. ${ }^{13}$ Sufficient blood for transfusion, autologous blood recovery system (Cell Saver), and staff should be secured, and discussions with the departments of anesthesiology, blood transfusion, urology, and obstetrics, as well as with the operating room staff, should be conducted in advance.

The positions of the fetus and placenta should be confirmed by ultrasonography, allowing the location of the uterine incision to be simulated. If total hysterectomy is scheduled for placenta previa increta or percreta, preoperative ureteral stent or hemostatic intra-arterial balloon occlusion catheter placement should be considered. ${ }^{1}$

\section{Tips and Warnings \\ Enhancement of Cooperation with Health Care Professionals}

In-hospital cooperation and hospital-clinic cooperation are important for prompt and appropriate patient transport and treatment. Discussions of the actions and measures against massive hemorrhage and obstetric emergencies should be made on a regular basis not only within the department of obstetrics but also with the departments of anesthesiology, clerical work, nursing, blood transfusion, and critical care, and necessary actions should be simulated within the hospital. It is necessary to understand actions to be taken for critical hemorrhage and not crossmatched compatible red blood cell (RBC) such as type $O$ RBC and type $A B$ fresh-frozen plasma (FFP), allowing consensus among the departments concerned to be reached. ${ }^{14}$ Cooperation between primary medical facilities and secondary and tertiary facilities should be deepened. Feedback regarding cases should be provided to facilities from which patients are transferred through case conferences or other suitable occasions. ${ }^{1,2}$

\section{Explanation of Procedures}

\section{Laparotomy}

A transverse skin incision may be used. If the field of view is narrow, the Maylard incision should be combined with the Pfannenstiel incision. In cases of intrauterine infection or rupture of the membranes, the use of a wound protector (Alexis) after laparotomy reduces the occurrence of abdominal wall wound disruption and wound infection. The peritoneum of the vesicouterine pouch is incised transversely and then the bladder is gently dissected.

When there are many engorged blood vessels in the surface of the myometrium or in the pericystic area, incision in the peritoneum of the vesicouterine pouch and separation of the bladder should be avoided ( - Figs. 5 and $\mathbf{6}$ ). Making a transverse incision, an oblique incision, or a J-shaped incision in the uterine corpus is recommended, that is, preoccupation with a transverse incision on the lower uterine segment is unwarranted.

\section{Tips and Warnings}

Contraindications of Placenta Manual Removal

The placenta should never be separated when engorged blood vessels are found in the area of placental 


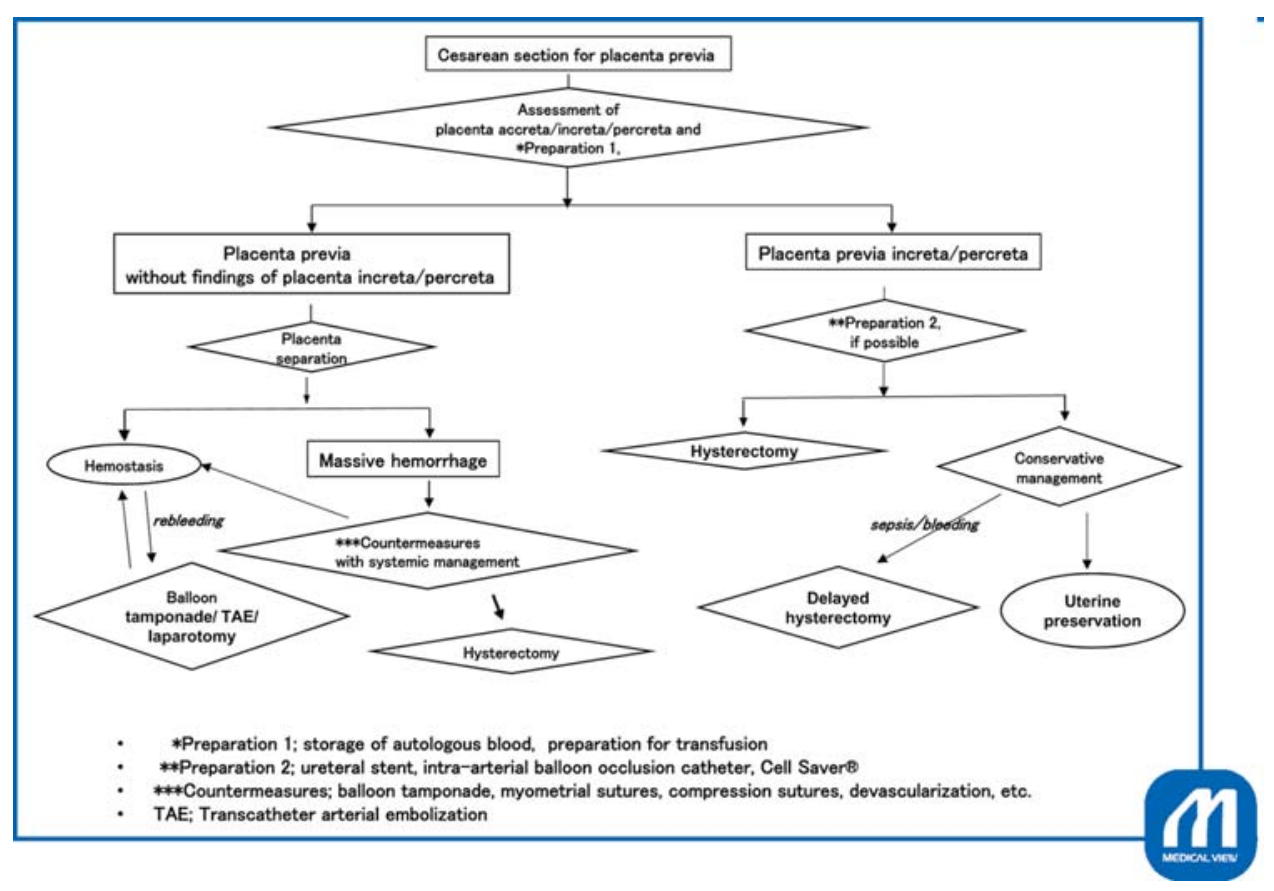

Fig. 4 Surgical strategies for placenta previa accrete spectrum and its management.

Table 1 Diagnostic methods and its findings of placenta accreta spectrum

\begin{tabular}{|l|}
\hline$\bullet$ Ultrasonography \\
\hline$\circ$ The presence of many low-intensity areas (placental lacunae) in the placenta; a Swiss cheese-like appearance. \\
\hline$\circ$ Loss of the low-intensity area (sonolucent zone, retroplacental clear space) between the placenta and myometrium. \\
\hline$\circ$ Irregularity and interruption of the bladder border. \\
\hline$\circ$ Thinning ( $\leq 1 \mathrm{~mm}$ ) of the myometrium, protrusion of the placenta toward the bladder. \\
\hline - Color-Doppler and pulse-Doppler ultrasonography \\
\hline$\circ$ There are diffuse blood vessels (lacunar flow) in the placenta with pulsating rapid venous flow. \\
\hline$\circ$ There are myometrial or subplacental blood vessels with pulsating turbulent venous flow. \\
\hline$\circ$ There are dilated blood vessels in the bladder and myometrium with arterial flow showing a low resistance index. \\
\hline$\circ$ Markedly dilated subplacental blood vessels with pulsating venous flow. \\
\hline$\bullet$ Biomarker testing \\
\hline$\circ$ Elevated $\alpha$-fetoprotein levels. \\
\hline$\circ$ Cell-free fetal DNA, placental mRNA, DNA microarray assay. \\
\hline
\end{tabular}

Source: Reproduced with permission of Takeda S. Cesarean section for placenta previa and placenta previa accrete spectrum. In: Takeda S, Hiramatsu Y, Konishi I, Sakuragi N, eds. OGS NOW, No.3. Cesarean Section. Mastering the Essential and Practical Surgical Procedures. Tokyo: Medical View; 2010:102-115. Copyright @ Medical View.

attachment to the anterior wall of the uterus with placental blood flow visible after laparotomy and when a diagnosis of placenta percreta or placenta increta has been made (-Fig. 6). ${ }^{1}$ Not only primary hysterectomy but also the conservative therapy or delayed hysterectomy (the two-stage hysterectomy) should be considered.
Incision of the Lower Uterine Segment

To avoid excessive splitting, it is recommended that a knife be used for myometrial incision, followed by the use of Cooper scissors to add any necessary incisions after the maternal surface of the placenta is observed. In reference to the placental location determined by preoperative ultrasonography, the incision should be extended in the direction that reaches the amniotic cavity by the shortest path, and the maternal surface of the placenta should be pressed 


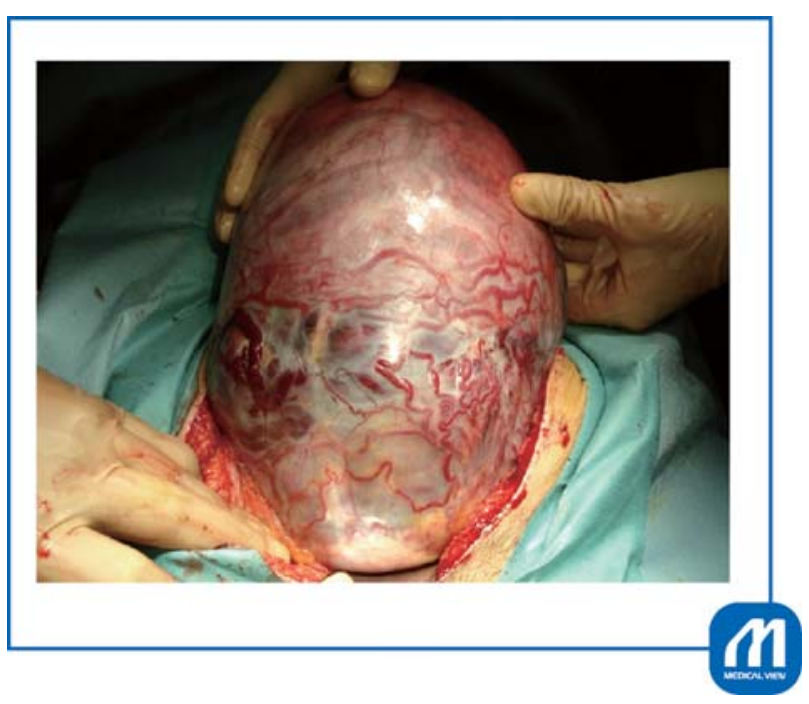

Fig. 5 Findings of the anterior wall of the uterus in placenta previa increta. The site of placental attachment to the anterior wall of the uterus is purpuric, and placental blood flow is seen through the wall. It is apparent that there are many engorged blood vessels, and that the placenta invades the area just beneath the serosa. No incision should be made around this area at the time of delivery of the fetus. A transverse incision in the uterine body, a J-shaped incision, or a transverse incision in the uterine fundus should be made at a site sufficiently distant from the placenta. (Reproduced with permission from Takeda S. Cesarean section for placenta previa and placenta previa accrete spectrum. In: Hiramatsu Y, Konishi I, Sakuragi N, Takeda S, eds. Mastering the Essential Surgical Procedures OGS NOW, No.3. Cesarean section. (Japanese). Tokyo: Medical View; 2010:102-115. Copyright $\odot$ Medical View).

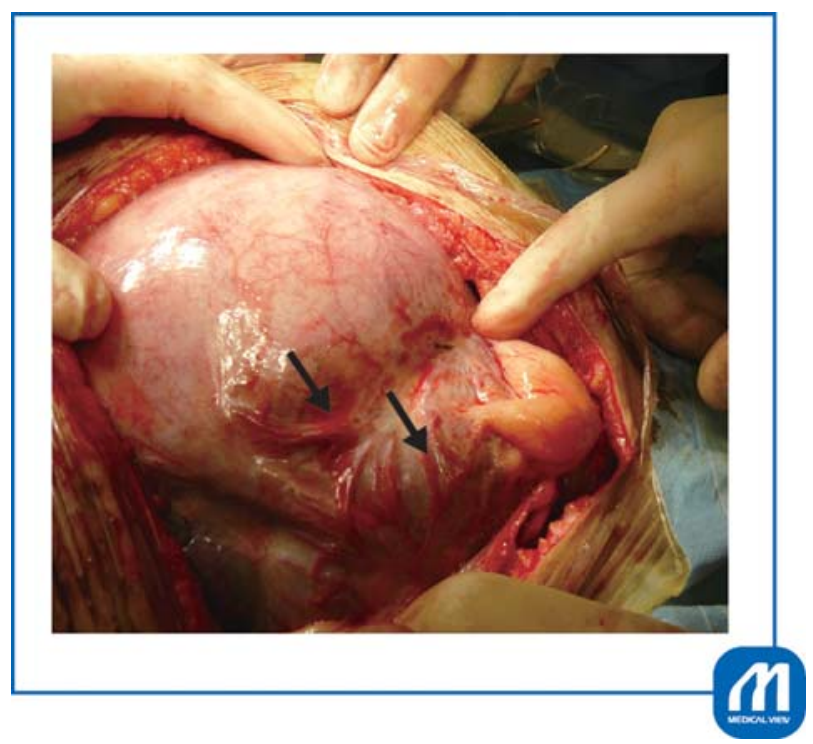

Fig. 6 Placenta previa with previous cesarean section. Because of a prior cesarean section, the bladder is elevated and adherent. Many blood vessels in the uterine surface around the incisional wound and in area around the bladder are engorged. $\rightarrow$ : There are engorged blood vessels in the bladder surface. (Reproduced with permission from Takeda S. Cesarean section for placenta previa and placenta previa accrete spectrum. In: Hiramatsu Y, Konishi I, Sakuragi N, Takeda S, eds. Mastering the Essential Surgical Procedures OGS NOW, No.3. Cesarean section. (Japanese). Tokyo: Medical View; 2010:102-115. Copyright (๑) Medical View). downward to expose the bulging fetal membranes. After rupture of the membranes, the fetus should be delivered while pressing the placenta downward while avoiding placental separation. It is not necessary to deliver the placenta first or to deliver the fetus transplacentally. In placenta previa cases in which the placenta is mainly attached to the anterior wall, the myometrial incision should be cut upward toward the right or left amniotic cavity, or a longitudinal incision in the uterine corpus may be made in an area where there is no placenta.

\section{Delivery of the Fetus}

The fetus should be delivered promptly after the rupture of the membranes, and the umbilical cord should be clamped to prevent anemia of the neonate. At the same time, the first assistant should hold the bleeding site as well as both ends of the uterine incision on the right and left sides with serrated forceps (At least eight pairs of the forceps should be placed on the operating table; each pair should be disinfected and ready for additional use) to achieve hemostasis to the maximum extent possible. When there is massive hemorrhage, the uterine incisional wound located downward may be unobservable and not possible to hold. In this case, the lower myometrial cut end of one lateral end of the uterine wound should be held and elevated with serrated forceps while aspirating blood, and the site of clamping should gradually be shifted to the center by alternating the forceps.

When hemorrhage is severe, pressure hemostasis should be employed with two or three rolled towels placed on the placental separation surface. A uterotonic agent should be administered locally and intravenously to achieve uterine contraction. When bleeding from the placental separation surface is severe, direct $Z$ suture with 1-0 synthetic absorbable thread, or $\mathbf{U}$-shaped suture or enclosing suture interrupted suture piercing the myometrium, should be performed. ${ }^{1}$ If these procedures fail to achieve hemostasis, compression sutures such as a vertical suture ${ }^{15,16}$ or an arterial ligation should be used. If hemorrhage is minor, gauze packing or uterine balloon tamponade is performed ( - Fig. 7); the gauze or balloon is removed transvaginally the following day. ${ }^{16}$

\section{Closure of the Uterine Wound and the Abdomen}

The right and left ends of the myometrial incisional wound should be sutured by interrupted or Z-suture, making sure that the thread is exposed to the uterine cavity. Caution is necessary because the inner surface may be deeply cleaved. The incisional wound is then sutured by interrupted suture with synthetic absorbable thread, while keeping the endometrial surface consistent. A thick myometrium may be sutured in two layers. Even when the bladder is separated, it is better not to suture the vesicouterine pouch; the bladder may otherwise be elevated. After intraperitoneal irrigation with physiological saline, the hemostatic status is confirmed, and a closed low-pressure continuous drain is inserted into the Douglas pouch and the site of myometrial suture. Absorbable adhesion barrier, such as Seprafilm is applied to the wounds on the uterus. 


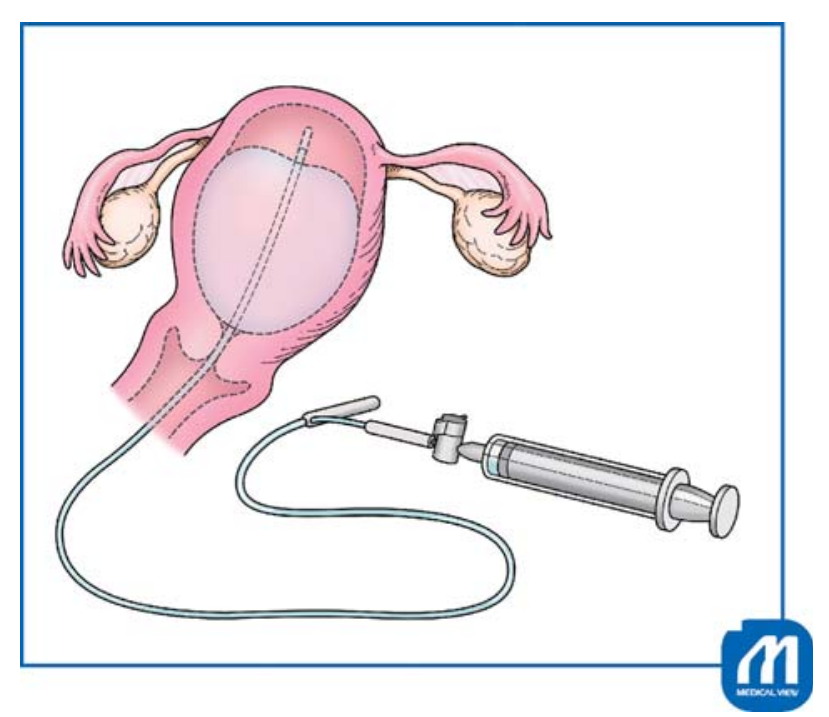

Fig. 7 Intrauterine balloon tamponade. If there is postoperative bleeding from the surface of placenta previa separation or due to atonic bleeding, intrauterine balloon tamponade should be attempted. Bakri, Fuji-metro, etc., balloons are available. After infusing physiological saline, in quantities of 80 to $200 \mathrm{~mL}$, into the balloon, gauze should be packed in the vagina to prevent the balloon from dislodging. (Reproduced with permission from Takeda S. Cesarean section for placenta previa and placenta previa accrete spectrum. In: Hiramatsu Y, Konishi I, Sakuragi N, Takeda S, eds. Mastering the Essential Surgical Procedures OGS NOW, No.3. Cesarean section. (Japanese). Tokyo: Medical View; 2010:102-115. Copyright @ Medical View).

The fascia should be sutured by continuous suture with a monofilament loop thread such as PDSII. Before performing subcutaneous suture, the subcutaneous wound should be irrigated with physiological saline, followed by abdominal closure. If the subcutaneous tissue is thick, subcutaneous suture or dermal suture with closed subcutaneous drain placement, should be performed, and the skin should be closed with tape.

\section{Tips and Warnings}

\section{Measures for Uterine Hemorrhage after Cesarean} Section

Uterine hemorrhage following abdominal closure after cesarean section may be due to bleeding from the surface of placenta previa separation, atonic bleeding, bleeding from the site of myometrial suture, or uterine hemorrhage due to disseminated intravascular coagulation (DIC). If the bleeding is from the surface of placenta previa separation, a hemostatic procedure using balloon tamponade is effective. ${ }^{16}$ In facilities where arterial embolization is feasible, embolization of the uterine artery or the internal iliac artery is indicated. In DIC cases, supplementation of coagulation factors and platelets, along with these measures, is important. ${ }^{16-18}$ If the above procedures have failed to control bleeding, laparotomic hemostasis or total hysterectomy should be performed without hesitation.

\section{Cesarean Section for Placenta Previa Increta/Percreta}

\section{Surgical Steps}

1. Ureteral stent insertion and arterial balloon occlusion catheter placement $\downarrow$

2. Laparotomy and observation whether or not there are engorged blood vessels in the area of placental attachment to the anterior wall of the uterus, with placental blood flow visible as findings of placenta previa increta or percreta

$\downarrow$

3. Incision on the uterine myometrium apart from placental attachment

$\downarrow$

4. Delivery of the fetus and myometrial suture $\downarrow$

5. Initiation of hysterectomy or abdominal closure (conservative treatment while leaving the placenta in situ or delayed two-stage hysterectomy) $\downarrow$

6. Hysterectomy: Cutting of the round ligament, incision, and exposure of the broad ligament $\downarrow$

7. Cutting of the ovarian ligament and the fallopian tube

$\downarrow$

8. Incision in the posterior lobe of the broad ligament and confirmation of the ureter $\downarrow$

9. Separation and cutting of the connective tissue around the cardinal ligament, manipulation of uterine blood vessels, cutting and suture of the sacrouterine ligament $\downarrow$

10. Cutting and suture of the cardinal ligament $\downarrow$

11. Separation of the bladder after interruption of the uterine blood flow. The bladder located inferior to the site of adhesion is separated first from the lower uterine segment and the vaginal canal (Pelosi $\operatorname{method}^{19}$ ) $\downarrow$

12. Cutting and closure of the vaginal canal $\downarrow$

13. Drain insertion and abdominal closure

\section{Approaches to Reduce Intraoperative Blood Loss}

\section{Tips and Warnings}

Placenta previa and placenta previa accreta/increta/percreta are known to frequently be associated with 
implementation of hysterectomy after delivery. In connection with these conditions, maternal death due to massive hemorrhage, DIC, multiple organ failure, and the need for at least 20,000 to $30,000 \mathrm{~mL}$ of blood transfusion have been reported. ${ }^{1}$ Along with the increase in the rate of cesarean section, it appears that the incidences of placenta previa and placenta previa accreta/increta/percreta will rise ever further in the future. Under the present situation in which placenta accreta and partial placenta accreta cannot be diagnosed accurately, it is necessary to be well versed in hemostasis by the balloon occlusion catheter technique, various methods of suture hemostasis, and total hysterectomy procedures.

\section{Intra-arterial Balloon Occlusion}

Temporary intra-arterial balloon occlusion catheters have come to be used to avoid massive hemorrhage in cases of placenta previa accrete spectrum with previous cesarean section. ${ }^{20-22}$ Because it is not possible to accurately diagnose placenta accreta spectrum, particularly placenta accreta and partial placenta accreta/increta/percreta, measures against bleeding should be prepared in advance. It is extremely difficult to control bleeding after massive hemorrhage occurs, and ligation of the internal iliac artery by exposing the retroperitoneal space and arterial clamping requires experience.

Therefore, it is useful to place an intra-arterial occlusion catheter in the common iliac artery or the abdominal aorta preoperatively; once bleeding occurs, the balloon will be inflated to block blood flow (-Fig. 8). The balloon may be placed in the aorta, common iliac artery, internal iliac artery, etc., but it has occasionally been reported that the placement in the internal iliac artery is ineffective because of the abundant anastomosis between the external and internal iliac arteries. ${ }^{23}$ The aorta and the common iliac artery are recommended as the sites of catheter placement $\left(\mathbf{- F i g . ~ 9 ) .}{ }^{20}\right.$

\section{Other Approaches for Reducing Bool Loss}

- The internal iliac arteries should be freed to allow clipping.

- Nelaton catheter should be passed through the bilateral broad ligament under the lower segment of the uterus to make the lower uterine segment ischemic by tying.

- Arterial ligation or local hemostasis such as $\mathbf{U}$-shaped sutures, enclosing sutures, an interrupted circular suture, compression sutures, and balloon tamponade, were reported.

However, these methods are not so effective for the prevention of massive hemorrhage, especially during the separation of the bladder, in cases with placenta previa percreta. Recently, embolization of the bleeding artery in the patient undergoing surgery for placenta previa percreta was reported in a hybrid operating room equipped with a fluoroscopic apparatus. This method might be useful for patients in whom various local hemostatic procedures are ineffective, if it is available.

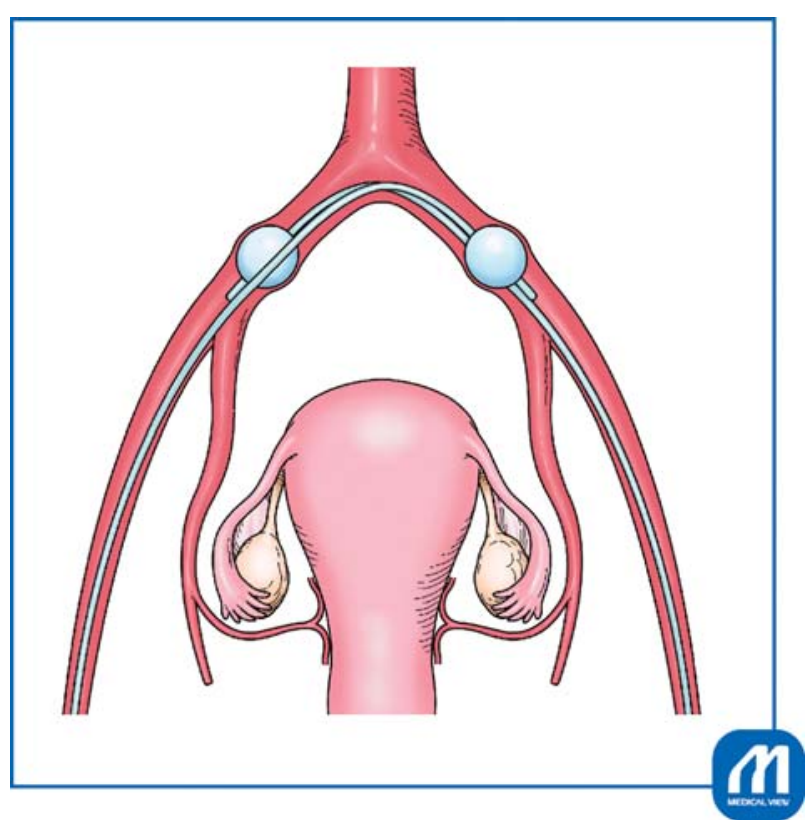

Fig. 8 Common iliac artery balloon occlusion. An occlusion catheter should be inserted from the right or left femoral artery to the contralateral common iliac artery by means of Seldinger arteriography, and how many milliliters of solution balloon inflation are required to block blood flow should be checked. Likewise, another occlusion catheter should be inserted from the contralateral femoral artery to cross the preceding catheter. The catheters should necessarily be crossed because, if this is not done, the balloons may be dislodged by arterial pressure during inflation. Inflation of a balloon will not achieve complete blockage of blood flow of the artery because there is also a contralateral catheter. Heparin 3,000 to 5,000 units should be infused intravenously before inflation of the balloons to prevent arterial thrombus. Time of blockage should be at least 40 minutes, possibly as much as 60 minutes. If longer blockage is necessary, blood flow should be released once and then blocked again. Aortic balloon occlusion uses only a single catheter, and therefore requires a shorter time for catheter insertion. However, the range of blockage is greater, and the catheter is thicker. In the gravid uterus, there is abundant anastomosis of the peripheral vessels of the internal iliac artery, with inflow of blood from the external iliac artery and the lumbar artery. Therefore, the hemostatic effect of balloon occlusion of the internal iliac artery on uterine hemorrhage is inconspicuous. Recently, the intra-aortic balloon occlusion is more often used to prevent massive hemorrhage for cesarean section in a case with placenta accrete spectrum because of a single catheter. The balloon occlusion technique is applicable to gynecologic surgery; it is particularly useful for blockage of blood flow in cases of myomectomy for giant cervical myoma, etc. (Reproduced with permission from Takeda S. Cesarean section for placenta previa and placenta previa accrete spectrum. In: Hiramatsu Y, Konishi I, Sakuragi N, Takeda S, eds. Mastering the Essential Surgical Procedures OGS NOW, No.3. Cesarean section. (Japanese). Tokyo: Medical View; 2010:102-115. Copyright (c) Medical View).

\section{Explanation of Procedures}

\section{Preoperative Procedures}

When the diagnosis of placenta percreta or placenta increta is made preoperatively, an arterial balloon occlusion catheter (to be placed in the common iliac artery or the aorta) and a ureteral stent should be inserted prior to surgery. ${ }^{1,2}$

\section{Laparotomy and Delivery of the Fetus}

A midline incision in the lower abdomen is recommended to secure the field of view for hysterectomy and arterial 


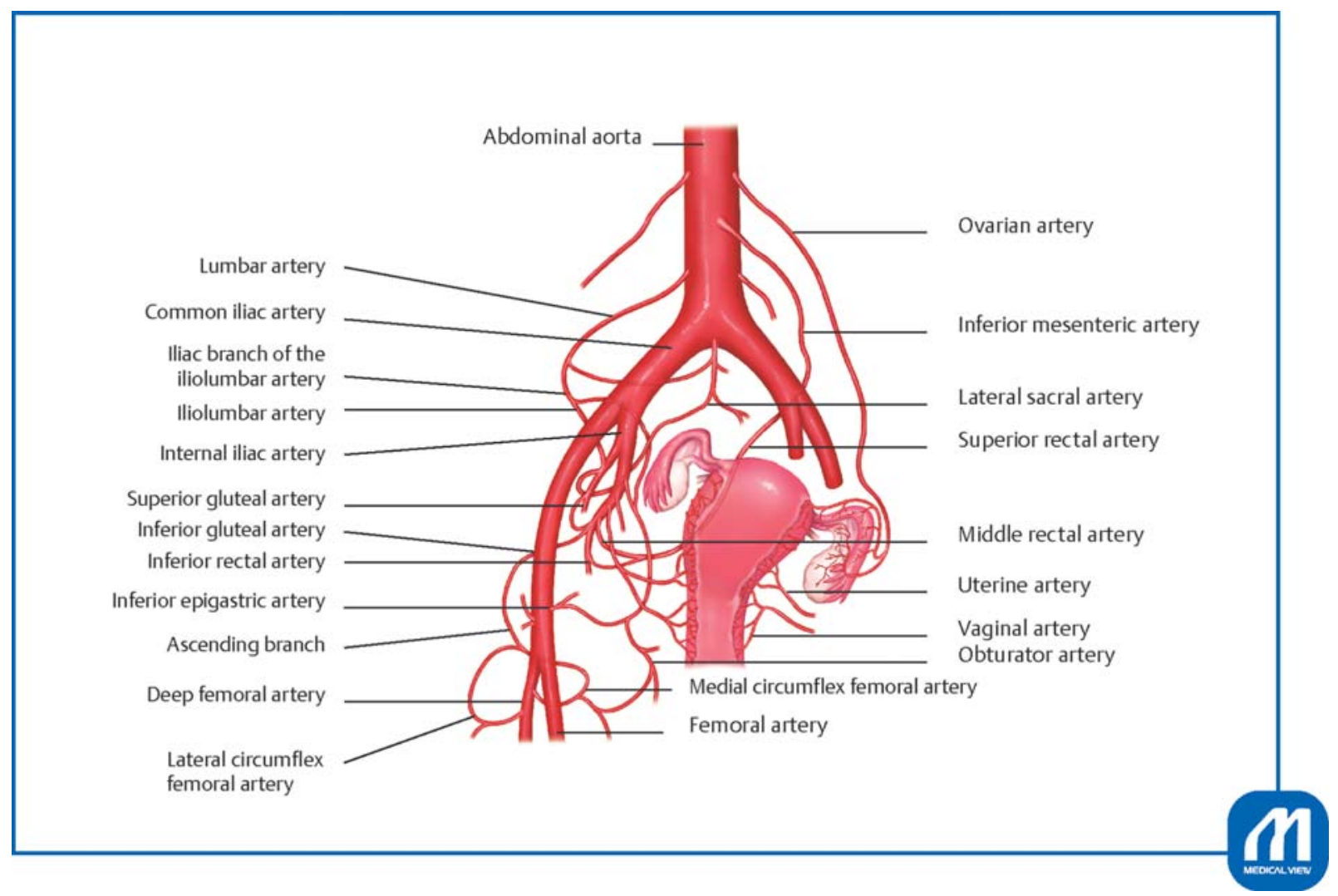

Fig. 9 Abundant pathways of collateral circulation to the uterus. Abdominal aorta. Lumbar artery. Common iliac artery. Iliac branch of the iliolumbar artery. Iliolumbar artery. Internal iliac artery. Superior gluteal artery. Inferior gluteal artery. Inferior rectal artery. Inferior epigastric artery. Ascending branch. Deep femoral artery. Lateral circumflex femoral artery. Ovarian artery. Inferior mesenteric artery. Lateral sacral artery. Superior rectal artery. Middle rectal artery. Uterine artery. Vaginal artery. Obturator artery. Medial circumflex femoral artery. Femoral artery (Reproduced with permission from Takeda S. Cesarean section for placenta previa and placenta previa accrete spectrum. In: Hiramatsu Y, Konishi I, Sakuragi N, Takeda S, eds. Mastering the Essential Surgical Procedures OGS NOW, No.3. Cesarean section. (Japanese). Tokyo: Medical View; 2010:102-115. Copyright $\odot$ Medical View).

ligation. When there are engorged blood vessels at the site of placental attachment to the anterior wall of the uterus, allowing placental blood flow to be visualized, or when a diagnosis of placenta percreta or placenta increta has already been obtained by diagnostic imaging, a transverse incision in the uterine body or in the uterine fundus ( - Fig. 10) should be made to prevent the incision from reaching the placenta and thereby causing massive hemorrhage. Rupturing of the membranes, delivery of the fetus, and cutting and ligation of the umbilical cord should be performed. Then, the myometrial wound should be temporarily closed.

\section{Cesarean Hysterectomy}

In routine cases, the operation proceeds to total hysterectomy. However, if there is no bleeding without spontaneous placental separation, it is also possible to provide conservative treatment with the placenta left in situ or to delay performing the hysterectomy.

In contrast to cases undergoing a routine cesarean hysterectomy, the placenta adherent to the areas from the uterine isthmus to the lower part of the uterine body shows a potbelly-like bulging, and many engorged blood vessels can be seen in areas around the bladder and around the scar from prior cesarean section. In some cases, the anterior surface of the uterus and the site of placental attachment are purple to dark red, and the placenta is translucent (-Fig. 11). The bladder is often elevated because of the prior cesarean section. For total hysterectomy, the operator should avoid vascular injury, ligating veins whenever possible, and pay attention to intraoperative uterine hemorrhage from the blood flow via the vagina and the bladder.

\section{Cutting of the Round Ligament and Incision and Exposure of} the Broad Ligament of the Uterus

The round ligament of the uterus and the ovarian ligament should be held bilaterally with a long straight Kocher clamp to elevate the uterus. After picking up the round ligament with Pean forceps, its medial side and lateral side should be ligated with absorbable thread, followed by cutting between the two. The lateral side should be processed by applying a double ligature. While pulling up the ligature, the peritoneum of the anterior lobe of the broad ligament should be incised in the directions toward the infundibulopelvic ligament and toward the bladder to expose the retroperitoneal space. At this time, the infundibulopelvic ligament should be traced cranially to identify the ureter. 


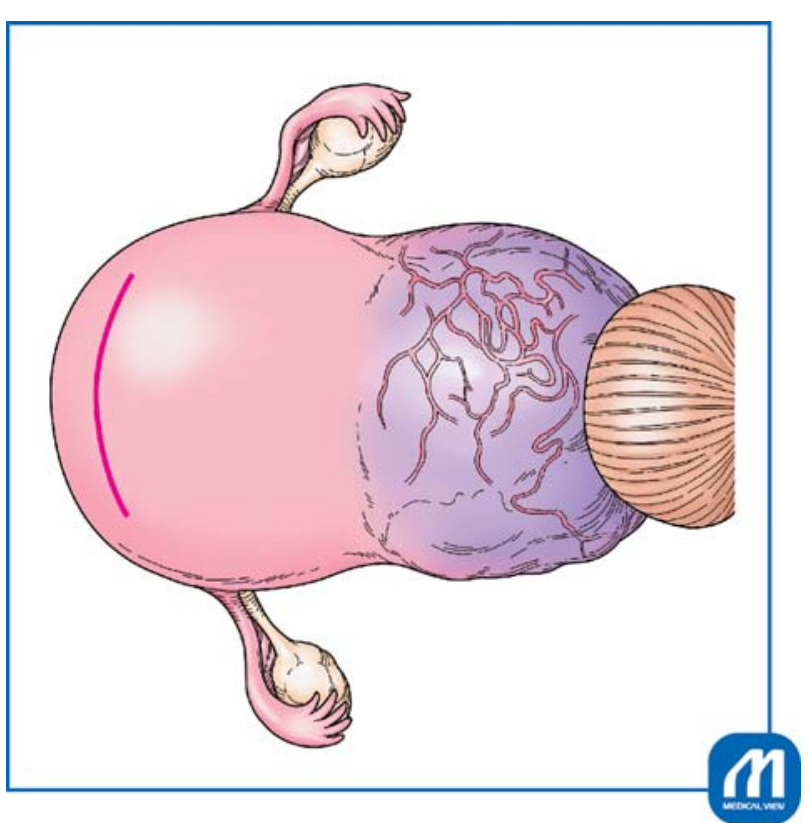

Fig. 10 Transverse incision in the uterine fundus (Kotsuji technique). A transverse incision is made in the anterior or posterior wall of the uterine fundus, avoiding the uterine horn and keeping a substantial distance from the area of placental attachment. When the incision reaches the uterine cavity, the myometrium is held with two Kelly clamps, and incised between the clamps to extend the incision. These Kelly clamps should be kept in place at this site until the fetus is delivered and the myometrium sutured. (Reproduced with permission from Takeda S. Cesarean section for placenta previa and placenta previa accrete spectrum. In: Hiramatsu Y, Konishi I, Sakuragi N, Takeda S, eds. Mastering the Essential Surgical Procedures OGS NOW, No.3. Cesarean section. (Japanese). Tokyo: Medical View; 2010:102-115. Copyright $\odot$ Medical View).

\section{Cutting of the Ovarian Ligament and the Fallopian Tube}

The peritoneum in the vicinity of the cut end of the round ligament should be incised in the direction toward the ovarian ligament, and the vascular plexus in areas around the tube and the ovary should be identified. A hole should be created and expanded with a Kelly clamp in the posterior lobe of the nonvascular broad ligament located inferior to the vascular plexus. The ovarian ligament and the tube should be clamped with serrated forceps and cut between the forceps on the uterine side. The lateral cut end should be sutured employing a figure-eight suture or interrupted suture. Because this procedure involves focused ligation, it is necessary to use the double ligature technique to prevent loosening. If the cut end is close to the suture thread, Kobayashi's ligation (vertical $\mathrm{Z}$ suture) ${ }^{24}$ should be added. When the cut end is broad, or when there are engorged blood vessels, the tube and the ovarian ligament should be ligated and cut separately. Recently tubectomy is performed for prophylaxis of the tubal origin ovarian cancer.

\section{Incision in the Anterior and Posterior Lobe of the Broad Ligament}

The course of the ureter should be carefully assessed in all cases. Such assessment is easy if a ureteral stent is in place. If adhesion is severe, and the peritoneum of the vesicouterine pouch is not clearly identifiable, it should be left untouched. The bladder should not be separated until the final stage.

\section{Cutting and Suturing of the Cardinal Ligament}

The connective tissue around the cardinal ligament should be separated and cut, followed by identification of uterine blood vessels, confirmation of the route of the ureter, and cutting and ligation of the ascending branches of the uterine artery and vein. Then, the sacrouterine ligament should be cut and sutured.

The lower part of the uterus shows a potbelly-like bulging; the cardinal ligament is broad and elongated, and, therefore, caution is necessary to avoid excessive cutting (-Figs. 10 and 11). Because the ureter is located extremely close to the uterus, the course of the ureter should be checked, or the stent should be confirmed. For cutting of the cardinal ligament (parametrium), the ligament should be clamped on the uterine side as well, to prevent bleeding on the uterine side. The cutting and suturing procedure of the cardinal ligament should be performed in at least three steps. Because the bladder is not yet separated, the cutting and suturing of the cardinal ligament should be advanced while separating the bladder and the connective tissue in the anterior portion of the cardinal ligament.

\section{Separation of the Bladder}

When there is bleeding around the bladder or massive uterine hemorrhage, blood flow should be blocked by the occlusion catheter as needed. If there is no bleeding, blockage of the blood flow is performed before separation of the bladder. Blockage should be maintained for not more than 40 minutes to 1 hour. If a blockage over an extended time period is necessary, blood flow blockage should be released once followed by reinstitution of the blockage.

If the blood flow of the common iliac artery or aorta (flow to the lower limbs) is to be blocked, unfractionated heparin 3,000 to 5,000 units (60-100 units $/ \mathrm{kg}$ ) should be infused intravenously in advance.

When separating the bladder, the site of placenta percreta and the site of adhesion should be left untouched, and the separating procedure should begin with a lower part of the bladder (Pelosi method). ${ }^{19}$ The space between the bladder and the lower uterine segment should be separated. After a penetration passage is formed like a tunnel between the right and left sides, the vaginal wall and the remaining parametrium should be held with serrated large curved forceps to block blood flow from the vagina (-Fig. 12).

When cutting the adhesion between the bladder and the uterus using an electric cautery tool or other equipment, it is helpful to elevate the bladder to add tension to the site of incision. In cases of complete placenta percreta with hematuria, opening the bladder lumen is helpful. Even if the placenta comes out of the uterine wall, bleeding inside the uterus is predominant, and therefore, the separating procedure should be performed without hesitation. Bleeding from the bladder separation surface is often severe, and the bleeding area should be sutured and reinforced with 3-0 absorbable thread in a covering manner. If the bladder lumen is open, double layer suture should be used. 


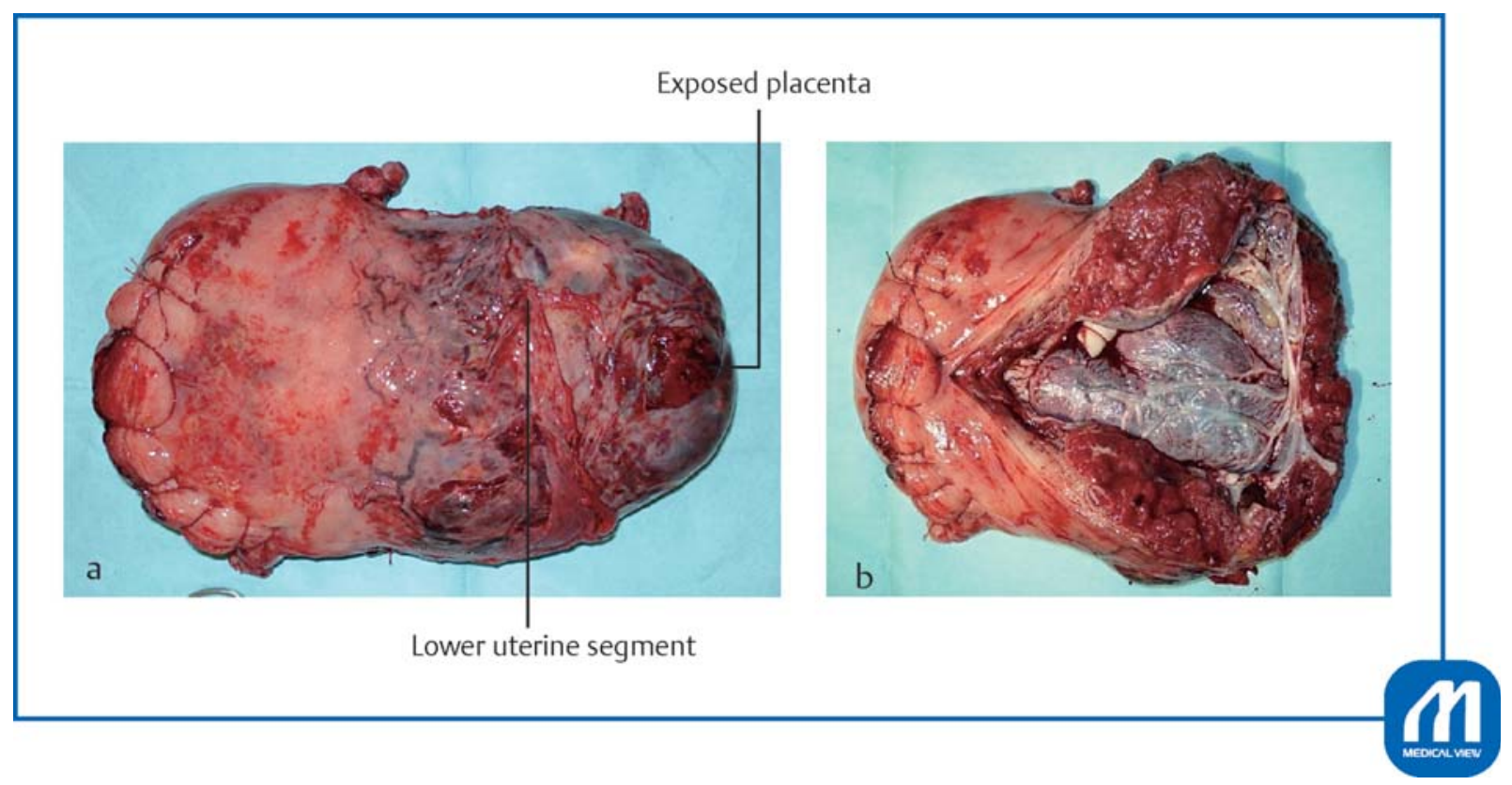

Fig. 11 Surgical specimen of the uterus with placenta percreta. The fetus was delivered by transverse incision in the uterine fundus. The lower part of the uterus was enlarged, showing a potbelly-like protrusion, in a broad range due to placental attachment. The ureter was located extremely close to the uterus. From the time of separation of the bladder, blood flow was blocked twice for 30 minutes with a balloon occlusion catheter to perform total hysterectomy. The placenta was exposed in the area of bladder separation, leading to a pathological diagnosis of placenta percreta. The amount of intraoperative bleeding was $1,500 \mathrm{~mL}$. Autologous blood alone, a quantity of $500 \mathrm{~mL}$, was transfused, and the surgery was completed. (a) Anterior uterine view; (b) Posterior uterine view. The posterior uterine walls were cut to open the uterine cavity. See the placenta to invade into the anterior uterine lower segment around the cesarean section scar. (Reproduced with permission from Takeda S. Cesarean section for placenta previa and placenta previa accrete spectrum. In: Hiramatsu Y, Konishi I, Sakuragi N, Takeda S, eds. Mastering the Essential Surgical Procedures OGS NOW, No.3. Cesarean section. (Japanese). Tokyo: Medical View; 2010:102-115. Copyright @ Medical View).

\section{Cutting and Closure of the Vaginal Canal}

After the bladder is separated, the anterior vaginal wall should be cut, and the uterus then excised. The vaginal wall should be closed by $Z$ suture or simple ligation. Because blood flow of the external iliac artery system via the cardinal ligament is extremely abundant, strict hemostasis is necessary.

\section{Drain Insertion and Abdominal Closure}

After intraperitoneal irrigation, a drain should be placed in the Douglas pouch. The pelvic peritoneum may be left open. An adhesion preventing material such as Seprafilm should be applied to the pelvic floor and under the abdominal wall, and the abdominal incision wound should then be closed.

\section{Special Occasions}

Delayed Hysterectomy (The Two-Stage Hysterectomy) In cesarean section cases, if the placenta does not separate spontaneously, and there is no bleeding, the surgery can be completed with the placenta left in situ without trying vigorously to separate it. The placenta may be separated at the second stage after blood flow into the placenta is reduced, or the amount of bleeding may be decreased by scheduled delayed hysterectomy. ${ }^{1,2}$ In these cases, embolization of the internal iliac artery or the uterine artery prior to the two-stage operation reportedly leads to a decrease in the amount of bleeding. ${ }^{5}$ Delayed hysterectomy has a drawback since, even if scheduled, it cannot be performed in cases in which the placenta has been separated, and hemorrhage has occurred, during cesarean section.

\section{Conservative Management of Placenta Increta/Percreta}

The uterus can be preserved under the conditions that the placenta is not separated, and there is no hemorrhage, during the cesarean section. After abdominal closure is performed with the placenta left in situ, spontaneous separation of the placenta can be anticipated. ${ }^{25,26}$ In any event, patients should be informed in advance of the risk of massive hemorrhage and complications such as sepsis due to infection.

\section{Obstetric Damage Control}

Hemostasis often cannot be achieved promptly in cases of massive hemorrhage during cesarean section, accompanied by DIC. In such obstetric cases with DIC, particularly in the presence of hypothermia, acidosis, and vasopressor requirement, damage control surgery (DCS) and resuscitation, which represent the therapeutic concept of life-saving intervention for severe trauma should be performed because ordinary hemostatic procedures such as sutures, ligation, and coagulation, etc., are not effective and bleeding persists. ${ }^{17,18}$ To treat this condition, first, towel packing of the abdomen or the whole pelvis should be performed to provide pressure hemostasis as a part of the DCS, stopping surgical procedures, and pressing on the abdominal aorta temporarily by hands. Second, the patient should be warmed up to keep in appropriate body temperature and 


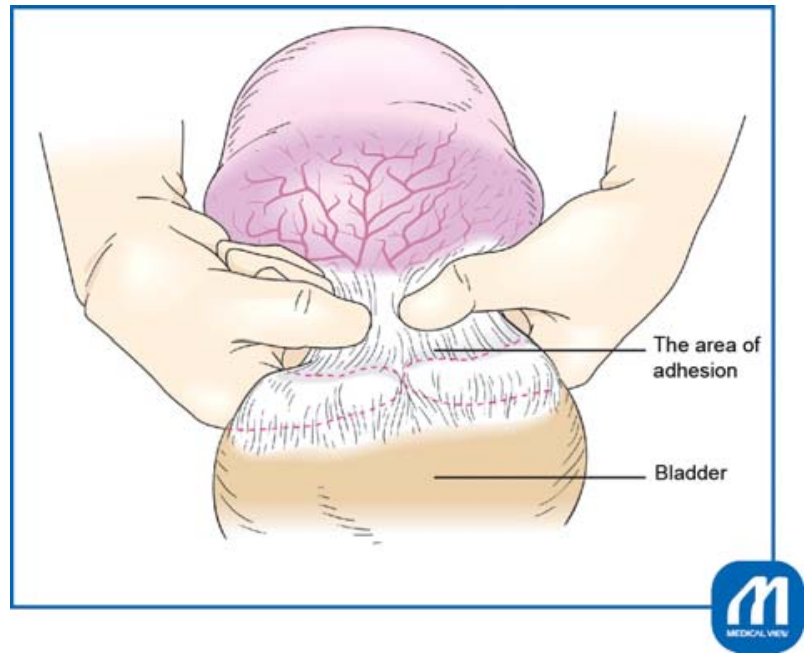

Fig. 12 Pelosi method. When blood flow cannot be blocked after a major part of the cardinal ligament is cut or when adhesions are extensive in placenta percreta cases, separation of the bladder should be performed in the last step. First, manual separation between the nonadherent vaginal wall under the placenta percreta and the lower part of the bladder should be performed to make a tunnel. The vaginal wall should be held with large curved forceps from the right and left sides, and the blood flow from the vagina should be blocked. Finally, the bladder is separated from the uterus. The area of adhesion. Bladder. (Reproduced with permission from Takeda S, Murayama Y. Cesarean hysterectomy for placenta previa accrete spectrum. In: Hiramatsu Y, Konishi I, Sakuragi N, Takeda S, eds. Mastering the Essential Surgical Procedures OGS NOW, No.9. Surgery for pregnancy with placenta previa and placenta accrete: Careful preparation and critical management. (Japanese). Tokyo: Medical View;

2012:122-133. Copyright (c) Medical View).

be treated with blood transfusion and "triple C supplement" such as combined administration of concentrated coagulation factors and FFP promptly to obtain a blood fibrinogen level of at least 150 to $200 \mathrm{mg} / \mathrm{dL}$ and to resuscitate the patient from shock and DIC and to prevent maternal death. $^{17}$

If possible, intraoperative insertion of intra-aortic balloon occlusion catheter could be another choice. If coagulopathy is eliminated, the conventional hemostatic procedures become effective. Thus, implementation of resuscitation while the surgical procedure is suspended allows avoidance of hemorrhagic death. ${ }^{16-18}$

\section{Treatment for Massive Hemorrhage and DIC}

Massive hemorrhage during delivery and total hysterectomy to control bleeding occur far more frequently during cesarean section than during vaginal delivery. Therefore, it is extremely important for obstetricians to learn appropriate and prompt responses to massive hemorrhage and DIC. $^{14,16-18}$

It is necessary to make arrangements for securing of staff, division of roles, access to the venous system, blood transfusion, testing, cross-matching, whole body management, hemostatic procedures, recording data, etc., all to be conducted in parallel. Communication among doctors, nursing staff, and clerical workers on a regular basis is impor- tant. The results of blood typing and irregular antibody testing (typing and screening) at the time of health checkups for pregnant women are useful for emergency transfusion. If there are no irregular antibodies, the physiological saline method is usually used for cross-matching tests. However, in critical massive hemorrhage cases, blood of the same $\mathrm{ABO}$ group is used, omitting the cross-matching test. If the blood of the same $A B O$ group is insufficient, non crossmatched compatible RBC such as type $O$ RBC and type AB FFP may be used. Infusion of approximately 15 units of FFP is required for raising the fibrinogen level by $100 \mathrm{mg} / \mathrm{dL}$. Transfusion of RBC and FFP should be performed to achieve the goals of hemoglobin 7 to $8 \mathrm{~g} / \mathrm{dL}$ or more, prothrombin time $70 \%$ or more, fibrinogen $150 \mathrm{mg} / \mathrm{dL}$ or more, and total protein $4.0 \mathrm{~g} / \mathrm{dL}$ or more. The platelet concentrate should be maintained at $100,000 / \mathrm{mm}^{3}$ or more.

\section{Simulation Training for Obstetric Emergency}

It is important to be familiar with emergency care and transfusion for obstetrical critical hemorrhage and to run a simulation of the preparation and actions to be taken in emergency settings. Such simulation training as the Japan Council for Implementation of Maternal Emergency LifeSaving System (J-CIMELS) training should involve the whole hospital, including not only the obstetrical team consisting of medical and paramedical staff members but also the clerical personnel in charge of the arrangement of blood transfusion, human resources, transfer of patients, and so on. Implementation of training and realistic simulations on a routine basis in the actual clinical setting is extremely important for ensuring prompt and appropriate responses to emergency cases. $^{27}$

\section{References}

1 Takeda S. Cesarean section for placenta previa and placenta previa accrete spectrum. In: Takeda S, Hiramatsu Y, Konishi I, Sakuragi N, eds. OGS NOW,No.3. Cesarean Section. Mastering the Essential and Practical Surgical Procedures. Tokyo: Medical View; 2010: 102-115

2 Takeda S, Murayama Y. Cesarean hysterectomy for placenta previa accrete spectrum. In: Konishi I, Hiramatsu Y, Sakuragi N, Takeda S, eds. OGS NOW,No.9. Surgery for Pregnancy with Placenta Previa and Placenta Accrete: Careful Preparation and Critical Management. Tokyo: Medical View; 2012:122133

3 Committee on Obstetric Practice. ACOG committee opinion: placenta accrete. Number 266, January 2002. American College of Obstetricians and Gynecologists. Int J Gynaecol Obstet 2002;77 (01):77-78

4 Oppenheimer L; Maternal Fetal Medicine Committee. Diagnosis and management of placenta previa. J Obstet Gynaecol Can 2007; 29(03):261-266

5 Sumigama S, Itakura A, Ota $\mathrm{T}$, et al. Placenta previa increta/ percreta in Japan: a retrospective study of ultrasound findings, management and clinical course. J Obstet Gynaecol Res 2007;33 (05):606-611

6 Jauniaux ERM, Alfirevic Z, Bhide AG, et al. Placenta praevia and placenta accreta: diagnosis and management. RCOG Green-top Guideline No. 27a. BJOG 2018. Available at: https://www.rcog. org.uk/en/guidelines-research-services/guidelines/gtg27a. Accessed December 7, 2019 
7 Oyelese Y, Smulian JC. Placenta previa, placenta accreta, and vasa previa. Obstet Gynecol 2006;107(04):927-941

8 Finberg HJ, Williams JW. Placenta accreta: prospective sonographic diagnosis in patients with placenta previa and prior cesarean section. J Ultrasound Med 1992;11(07):333-343

9 Thia EWH, Lee SL, Tan HK, Tan LK. Ultrasonographical features of morbidly-adherent placentas. Singapore Med J 2007;48(09): 799-802, quiz 803

10 Chou MM, Ho ESC, Lee YH. Prenatal diagnosis of placenta previa accreta by transabdominal color Doppler ultrasound. Ultrasound Obstet Gynecol 2000;15(01):28-35

11 Palacios Jaraquemada JM, Bruno $\mathrm{CH}$. Magnetic resonance imaging in 300 cases of placenta accreta: surgical correlation of new findings. Acta Obstet Gynecol Scand 2005;84(08):716-724

12 Berkley EM, Abuhamad A. Imaging of placenta accreta spectrum. Clin Obstet Gynecol 2018;61(04):755-765

13 Kuromaki K, Takeda S, Seki H, Kinoshita K, Maeda H. Indication and efficacy of autologous blood transfusion for pregnant women. J Obstet Gynaecol Res 2002;28(03):182-183

14 Takeda S, Makino S, Takeda J, et al. Japanese clinical practice guide for critical obstetrical hemorrhage (2017 revision). J Obstet Gynaecol Res 2017;43(10):1517-1521

15 Makino S, Tanaka T, Yorifuji T, Koshiishi T, Sugimura M, Takeda S. Double vertical compression sutures: a novel conservative approach to managing post-partum haemorrhage due to placenta praevia and atonic bleeding. Aust N Z J Obstet Gynaecol 2012;52 (03):290-292

16 Takeda S, Takeda J, Makino S. A minimally invasive hemostatic strategy in obstetrics aiming to preserve uterine function and enhance the safety of subsequent pregnancies. Hypertens Res Pregnancy 2019;7(01):9-15

17 Takeda J, Makino S, Takeda S. Hemostasis for massive hemorrhage during cesarean section. In: Schmolzer G, ed. Cesarean Delivery. IntechOpen; 2019. In press. DOI: 10.5772/intechopen.86394
18 Takeda J, Takeda S. Management of disseminated intravascular coagulation associated with placental abruption and measures to improve outcomes. Obstet Gynecol Sci 2019;62(05):299-306

19 Pelosi MA III, Pelosi MA. Modified cesarean hysterectomy for placenta previa percreta with bladder invasion: retrovesical lower uterine segment bypass. Obstet Gynecol 1999;93(5 Pt 2):830-833

20 Takeda J, Makino S. Temporary arterial balloon occlusion for obstetrical field. In: Takeda S, Kuwatsuru R, eds. Gynecologic and Obstetric Prophylactic Hemostasis by Intra-arterial Balloon Occlusion. Singapore: Springer; 2018:33-39

21 Ono Y, Murayama Y, Era S, et al. Study of the utility and problems of common iliac artery balloon occlusion for placenta previa with accreta. J Obstet Gynaecol Res 2018;44(03):456-462

22 Sone M, Nakajima Y, Woodhams R, et al. Interventional radiology for critical hemorrhage in obstetrics: Japanese Society of Interventional Radiology (JSIR) procedural guidelines. Jpn J Radiol 2015;33(04):233-240

23 Iwata A, Murayama Y, Itakura A, Baba K, Seki H, Takeda S. Limitations of internal iliac artery ligation for the reduction of intraoperative hemorrhage during cesarean hysterectomy in cases of placenta previa accreta. J Obstet Gynaecol Res 2010;36 (02):254-259

24 Takeda S, Kikuchi I. Surgery for endometrial cyst. In: Hiramatsu Y, Konishi I, Sakuragi N, Takeda S, eds. OGS NOW,No.1 Incisions, Closures and Operations for Adnexal Lesions. Mastering the Basic Surgical Techniques. Tokyo: Medical View; 2010:126-133

25 Ueda Y, Kondoh E, Kakui K, et al. Serial magnetic resonance imaging of placenta percreta with bladder involvement during pregnancy and postpartum: a case report. J Obstet Gynaecol Res 2013;39(01):359-363

26 Sentilhes L, Kayem G, Silver RM. Conservative management of placenta accreta spectrum. Clin Obstet Gynecol 2018;61(04):783-794

27 Takeda S. Education and training approaches for reducing maternal deaths in Japan. Hypertens Res Pregnancy 2018;6:15-19 